\title{
Tratamento não farmacológico da hipertensão na atenção primária: Uma revisão integrativa
}

\author{
Non-pharmacological treatment of arterial hypertension in primary care: An integrative review \\ Tratamiento no farmacológico de la hipertensión arterial em atención primaria: Uma revisión
}

integradora

Recebido: 30/05/2021 | Revisado: 09/06/2021 | Aceito: 14/06/2021 | Publicado: 29/06/2021

\author{
Ana Júlia Ribeiro Costa \\ ORCID: https://orcid.org/0000-0002-2938-5544 \\ Universidade Ceuma, Brasil \\ E-mail: anajuliacostarc@gmail.com \\ Cobias Amorim Ghidetti \\ ORCID: https://orcid.org/0000-0002-9866-1557 \\ Universidade Ceuma, Brasil \\ E-mail: Cobias1998@gmail.com \\ Rayonara Franco Macedo \\ ORCID: https://orcid.org/0000-0002-5329-1986 \\ Universidade Ceuma, Brasil \\ E-mail: rayonaramacedo@hotmail.com \\ Janine Silva Ribeiro Godoy \\ ORCID: https://orcid.org/0000-0001-5587-0896 \\ Universidade Ceuma, Brasil \\ E-mail: janinesrg@gmail.com \\ Carla Araújo Bastos Teixeira \\ ORCID: https://orcid.org/0000-0002-7357-772X \\ Universidade Ceuma, Brasil \\ E-mail: carlinhateixeira@hotmail.com
}

\begin{abstract}
Resumo
Este estudo objetiva avaliar as formas de tratamento não medicamentoso em pacientes portadores da Hipertensão arterial na atenção primária, bem como sua eficácia. Método: Trata-se de uma Revisão Integrativa de literatura em que foi utilizado como recurso metodológico levantamento bibliográfico nas seguintes plataformas: Lilacs, Medline, CVSP, Scielo bem como a plataforma do Google Acadêmico, agregando estudos científicos abordados entre os anos de 2015 a 2020. Neste levantamento bibliográfico utilizaram-se os descritores: Hipertensão Arterial (Arterial hypertension), Tratamentos não farmacológicos (Non-pharmacological treatments) e Atenção Primária (Primary attention). Resultados e Discussão: Com base nos estudos utilizados nesta pesquisa, verificou-se que os tratamentos não farmacológicos têm uma importância valiosa para a terapia da hipertensão Arterial Sistêmica, refletindo inclusive na prevenção de agravos em pacientes com fatores de riscos e comorbidade. Conclusão: Permitiu-se compreender que os tratamentos não farmacológicos têm efeitos positivos, mas precisam de um acompanhamento continuado, levando em consideração o contexto social e as dificuldades pessoais de cada indivíduo.
\end{abstract}

Palavas-chave: Hipertensão; Educação em saúde; Atenção primária.

\begin{abstract}
Objective: Analyze the tools used in the non-drug treatment of systemic arterial hypertension in the context of primary care. Method: It is an Integrative Literature Review in which bibliographic survey was used as a methodological resource on the following platforms: Lilacs, Medline, CVSP, Scielo as well as the Google Scholar platform, adding scientific studies covered between the years 2015 to 2020. In this bibliographic survey carried out over the internet the descriptors were used: Arterial Hypertension, Non-pharmacological Treatments and Primary Care. Results and Discussion: Based on the studies used in this research, it was found that non-pharmacological treatments have a valuable importance for the therapy of systemic arterial hypertension, even reflecting the prevention of diseases in patients with risk factors and comorbidity. Conclusion: It was allowed to understand that non-pharmacological treatments have positive effects, but need continuous monitoring, taking into account the social context and personal difficulties of each individual.
\end{abstract}

Keywords: Hypertension; Health education; Primary health care. 


\begin{abstract}
Resumen
Objetivo: Este estudio tiene como objetivo evaluar las formas de tratamiento no farmacológico en pacientes con hipertensión arterial en atención primaria, así como su efectividad. Método: se trata de una Revisión de Literatura Integrativa que se utilizó como recurso metodológico, levantamiento bibliográfico en las siguientes plataformas: Lilacs, Medline, CVSP, Scielo y también la plataforma Google Academic, agregando los estudios científicos cubiertos entre los años 2015 a 2020 Este relevamiento bibliográfico utilizará los siguientes descriptores: hipertensión, tratamientos no farmacológicos y atención primaria. Resultados y Discusión: Con base en los estudios utilizados en esta investigación, se encontró que los tratamientos no farmacológicos son de valiosa importancia para el tratamiento de la Hipertensión Arterial Sistémica, incluida la prevención de enfermedades en pacientes con factores de riesgo y comorbilidades. Conclusión: es posible comprender que los tratamientos no farmacológicos tienen efectos positivos, más precisamente de seguimiento continuo, teniendo en cuenta el contexto social y las dificultades personales de cada indivíduo.
\end{abstract}

Palabras clave: Hipertensión; Educación para la salud; Atención primaria.

\title{
1. Introdução
}

As Doenças Cardiovasculares (DCs) representam um significativo agravo para a saúde. Dados da Organização Mundial de Saúde/Organização Pan-Americana de Saúde (OMS/OPAS, 2017) estimam que 17,7 milhões de pessoas vieram a óbito em decorrência das DCs representando 31\% das mortes em todo o mundo. Dentre elas, a Hipertensão Arterial Sistêmica (HAS) é a detentora dos maiores números de morbidade e mortalidade em virtude de suas consequências, como Insuficiência Cardíaca (IC), Acidente Vascular Encefálico (AVE), Infarto Agudo do Miocárdio (IAM), Doença Arterial Periférica (DAP), Doença Renal Crônica (DRC) e morte súbita (Malachias et al., 2016). A HAS é identificada como uma condição clínica multifatorial em que há a elevação sustentada de níveis pressóricos $\geq 140$ e/ou $90 \mathrm{mmHg}$, podendo ser agravada por fatores de risco, a exemplo da dislipidemia e causar Lesões em Órgãos Alvo (LOA) (Fiório et al, 2020).

A HAS caracteriza-se por ser uma doença crônica, silenciosa e como forma de contribuir no manejo das pessoas acometidas se utiliza uma classificação baseada em valores, na qual os níveis considerados normais para pressão arterial (PA) são de $\leq 120 / 80 \mathrm{mmHg}$. Superior a estes valores encontram-se três estágios de hipertensão, estes estágios chamam atenção para níveis pressóricos capazes de causar danos no organismo do paciente e também são utilizados para determinar a conduta inicial do profissional. Ademais, existe um estado de alerta identificado como pré-hipertensão, em que o indivíduo apresenta uma PA sistólica entre 121 e 139 e a diastólica entre 81 e 89 mmHg, assim é possível aplicar medidas de intervenção que podem ser determinantes para beneficiar o indivíduo e evitar que o mesmo evolua seu quadro para HAS (Malachias et al., 2017).

Existem classificações que não levam em consideração unicamente os valores, mas particularidades na apresentação da patologia. Nestas têm-se a hipertensão do avental branco, em que há a apresentação de valores anormais quando o paciente se encontra em um consultório, mas na medição realizada em residência, os valores se mostram normais; hipertensão mascarada, em que há o inverso do que ocorre na anterior, e os valores normais são encontrados no consultório, entretanto a PA é elevada nas medidas residenciais; e por último a pressão sistólica isolada, em que há valores aumentados na pressão sistólica com a diastólica dentro dos valores de normalidade. Vale ressaltar, que para definir um diagnóstico de HAS não se deve levar em consideração apenas as aferições realizadas em consultas, mas também as residenciais, sendo as mesmas executadas em condições ideais e, no mínimo, em duas ocasiões diferentes (Malachias et al., 2016).

Os fatores de risco para o desenvolvimento dessa doença podem ser modificáveis ou não. De acordo com a Malachias et al. (2017) os fatores de risco não modificáveis como a idade, a etnia, a genética e o gênero, os mais afetados por estes aspectos são, respectivamente, os idosos com mais de 60 anos, os negros e as mulheres. Há ainda os fatores modificáveis que são constituídos pelos hábitos de vida de cada indivíduo a exemplo do sedentarismo, obesidade, uso de tabaco, ingestão excessiva de bebidas alcoólicas e de sódio, mais comumente consumido por meio do sal de cozinha. Além disso, existem 
questões socioeconômicas que devem ser avaliadas, uma vez que escolaridade e poder aquisitivo podem tornar-se obstáculos no acesso a informações do âmbito da saúde, assim não dependem do indivíduo, mas de uma intervenção estatal.

A HAS ainda é tomada em um panorama social descrito como representação social. Estas configuram-se como a forma que o paciente e o médico encaram o diagnóstico de uma patologia crônica e sem cura. As representações sociais se dão a partir do momento que o indivíduo toma consciência da dimensão que seus hábitos sociais serão mudados, deixando para trás sua vida normal, frente a necessidade de um estilo de vida compatível com a patologia crônica que possui. As representações sociais não abrangem apenas o paciente, mas também a conduta do profissional diante do seu paciente, uma vez que muitas das instruções repassadas após o diagnóstico de hipertensão não são seguidas pelo enfermo. Assim, as representações sociais do paciente precisam ser interpretadas pelo profissional, para que, ao passar as instruções de tratamento, o paciente entenda e possa aderir ao novo estilo de vida que sua atual realidade requer (Scholze et al., 2019).

A análise dos dados epidemiológicos acerca da HAS desde os níveis mundiais até as regiões brasileiras, permite identificar números alarmantes de como essa patologia crônica acomete a população. Ressaltando ainda que não refletem a real situação, tendo em vista as dificuldades de encontrar dados atuais e fidedignos de prevalência e incidência, considerando os poucos estudos, sobretudo nos pequenos centros populacionais. Neste contexto a OMS, faz uma estimativa que até 2025 haverá um crescimento global de $60 \%$, com cerca de 7,1 milhões de mortes anuais. Vale ressaltar que na perspectiva mundial, o Canadá quintuplicou seus números em relação ao controle da HAS, entre 1992 a 2009, apresentando os melhores índices no que se refere a prevalência, diagnóstico, tratamento e controle, servindo de modelo para outros países (Dantas \& Roncalli, 2016; Malta et al., 2018).

Por outro lado, um estudo multicêntrico descrito no ano de 2017 incluiu 154 países e demonstrou um aumento significativo da elevação da pressão arterial sistólica (PAS) entre 1990 e 2015, indo de 1,87 bilhão para 3,47 bilhões de pessoas acometidas (Consolim-colombo et al., 2019). Quando se avalia os dados a nível nacional, os números não deixam de ser impactantes, em média 84 mortes por hora e 829 por dia e no ano de 2017 foram 302 mil, refletindo as mortes por doenças cardiovasculares em que o principal fator de risco é a HAS (Laboissière, 2019). De acordo com Santiago et al. (2019), a reunião de dados de determinadas cidades brasileiras indica que a HAS atinge em média 30\% dos adultos, valor este que representa 36 milhões de pessoas. Os dados do Departamento de Informática do Sistema Único de Saúde (DATASUS) coletados através do programa HIPERDIA, que vigorou de 2002 a 2013 - acerca da HAS na região nordeste, demonstram que, nos meses de janeiro a abril de 2013 (exceto o estado do Pernambuco que só dispunha dos meses de janeiro a março), observou-se uma média de 895,6 casos de hipertensão. No estado do Maranhão, verificou-se o quantitativo de 1085 casos em quatro meses (Brasil, 2013).

Embora os dados se mostrem preocupantes, grande parte da população desconhece sua condição patológica, visto que como mencionado a HAS em geral se apresenta assintomática. Estima que de 100 indivíduos hipertensos, em torno de 75 sabem que possuem a doença e aproximadamente, 50 indivíduos realizam o tratamento. Todavia, ao analisar dados epidemiológicos a respeito de quantos pacientes em tratamento apresentam a pressão controlada, o percentual chega, no máximo a 40\%. Em um quantitativo absoluto de 100 hipertensos apenas 25 apresentam uma pressão arterial controlada. Apesar de haver um grande número de medidas terapêuticas e uma vasta gama dessas dependerem unicamente do paciente, a adesão, ou mesmo a utilização adequada dessas está muito aquém do esperado. O tratamento medicamentoso quando inclui a administração de mais de um medicamento se torna ainda menos eficiente no quesito adesão, uma vez que muitos dos pacientes são idosos e alegam esquecer ou se confundir quanto aos comprimidos e horário de administração (Jardim, 2018; Gewehr et al., 2018).

A Atenção Primária é o ponto chave para a reduzir o número de hipertensos e aumentar o entendimento da população acerca da HAS. Segundo Santiago et al. (2019), a HAS é a condição clinica mais habitualmente encontrada na Atenção 
Primária. Isto porque esta é o local em que é feita a promoção e a prevenção à saúde, bem como o tratamento e acompanhamento dos casos. É um ambiente capaz de promover a igualdade no acesso dos mais diversos grupos sociais que precisam da saúde pública, sobretudo, no acompanhamento das enfermidades crônicas. Desta forma, quando bem explorado todas as ferramentas que essa porta de entrada pode fornecer à população, há a capacidade de evitar os agravos e consequências dos níveis pressóricos descompensados. Com isso, a Atenção Primária tem capacidade de melhorar a qualidade de vida do paciente e reduzir os riscos para doenças cardiovasculares, diminuindo, as internações e mortes, bem como os custos para o país (Oliveira et al., 2020).

Como demonstrada, a HAS além de um importante problema de saúde pública com repercussões relevantes para outras doenças cardiovasculares, também constitui uma patologia que, por conta de suas consequências, gera um alto custo para os serviços de saúde. No entanto, há a possibilidade de intervenção com medidas educacionais que objetivem explorar a prevenção e enfatizar os tratamentos não medicamentosos, por meios das mudanças nos hábitos de vida. Isto pode gerar reflexos nos diversos âmbitos da saúde, proporcionado uma diminuição da demanda de pacientes com complicações em virtude da HA, direcionando o contingente para a manutenção do controle e prevenção de agravos destes pacientes. Deste modo, este estudo objetiva avaliar as formas de tratamento não medicamentoso em pacientes portadores da HAS na Atenção Primária, bem como sua eficácia.

\section{Metodologia}

Este estudo trata de uma revisão integrativa produzida em quatro etapas: primeiro realizou-se a delimitação da pergunta norteadora, em um segundo momento a busca de artigos nas bases de dados, seguido pela análise e avaliação dos documentos encontrados. Depois foram separados os que seriam incluídos na revisão integrativa de acordo com a sua temática, com vista ao domínio de conteúdo acerca da questão norteadora "Quais as opções terapêuticas utilizadas para o tratamento não farmacológico de HAS na atenção primária?".

A coleta de dados foi efetuada em cinco bases de dados: Campus Virtual em Saúde Pública (CVSP), Google acadêmico, Literatura Latino-Americana e do Caribe em Ciências da Saúde (Lilacs), Medline e Scientific Electronic Library Online (Scielo), tendo como critério de inclusão serem artigos publicados entre 2015 a 2020. Foram selecionados artigos nas línguas portuguesa e em inglesa. Foram excluídos artigos de revisão, teses, dissertações e editoriais.

Para delimitação das buscas foram utilizados os descritores em português: Hipertensão, Tratamento não medicamentoso, atenção primária; e em inglês: Hypertension, Non-drug treatment, primary attention. Foram incluídos os artigos completos que contemplassem o assunto a ser estudado, disponibilizados de forma eletrônica e gratuito nas bases de dados que foram selecionadas.

Os critérios de seleção foram aplicados na plataforma, após isto foi realizada a busca por títulos nos artigos localizados. Um novo filtro foi feito, mas desta vez os artigos selecionados no filtro por títulos foram lidos na íntegra, e somente aqueles que tinham a temática com a perspectiva que contemplava a abordagem deste trabalho foram escolhidos. Por fim, uma nova leitura foi realizada dentro dos artigos finais para compor a tabela, e destes um foi excluído por haver desvio na abordagem central deste trabalho (Tabela 1).

No que concerne aos artigos selecionados para a tabela 2 foram realizadas as leituras completas dos mesmos e avaliados os conteúdos quanto a clareza das ideias e da apresentação dos métodos, a evidência dos resultados e a organização da tabela com o periódico, ano, país, tipo de estudo, identificação do autor, objetivo do estudo e conclusão. O tipo de estudo que caracteriza este artigo é do tipo qualitativo, Pereira et al (2018), define os métodos qualitativos como "aqueles nos quais é importante a interpretação por parte do pesquisador como usar opiniões sobre o fenômeno em estudo". 
Tabela 1 - Fluxograma da coleta de dados para revisão integrativa.

(Total: 11 artigos incluídos).

\begin{tabular}{lcccc}
\hline \multicolumn{1}{c}{$\begin{array}{c}\text { Bases de } \\
\text { dados }\end{array}$} & $\begin{array}{c}\text { Artigos } \\
\text { localizados }\end{array}$ & $\begin{array}{c}\text { Artigos } \\
\text { selecionados } \\
\text { pela leitura na } \\
\text { integra }\end{array}$ & $\begin{array}{c}\text { Artigos selecionados } \\
\text { para compor o } \\
\text { quadro }\end{array}$ & $\begin{array}{c}\text { Artigos } \\
\text { excluidos }\end{array}$ \\
\hline CVSP & 17 & 0 & 0 & 17 \\
Google & 94 & 2 & 2 & 92 \\
Acadêmico & 58 & 14 & 5 & 53 \\
Lilacs & 55 & 9 & 1 & 54 \\
Medline & 10 & 5 & 3 & 7 \\
Scielo & $\mathbf{2 3 4}$ & 30 & $\mathbf{1 1}$ & 223 \\
Total & & & & \\
\hline
\end{tabular}

Fonte: Autores.

\section{Resultados e Discussão}

A amostra final desta revisão foi constituída por onze documentos: dez artigos científicos e uma diretriz internacional com formato de artigo, selecionados do montante de 234 documentos pelos critérios de inclusão previamente estabelecidos. A Tabela 1 apresenta o total de artigos encontrados, selecionados e inclusos de cada base de dados, esta é constituída por ano, título, autoria, objetivo e conclusão.

Na base de CVSP foram encontrados 17 artigos, dos quais nenhum foi selecionado para a segunda triagem, tendo em vista que dez possuíam inconformidades, dois eram do tema, mas não eram artigos, quatro sem acesso (necessitavam de senha ou precisavam ser pagos) e um era Trabalho de Conclusão de Curso (TCC). No Google Acadêmico foram encontrados 94 artigos dos quais noventa e dois foram excluídos, pois quatro não abordavam a temática farmacológica, cinquenta e três possuíam inconformidades, três sem acesso e trinta eram TCC ou monografias, assim, restaram dois para compor a tabela.

$\mathrm{Na}$ plataforma Lilacs foram encontrados 58 artigos dos quais quarenta e quatro foram excluídos na primeira triagem, destes, catorze não abordavam a temática não farmacológica, vinte e nove possuíam inconformidades e um não tinha acesso. Dos catorze artigos que haviam passado para leitura na integra, ficaram apenas cincos que contemplavam a perspectiva da pergunta norteadora, os demais foram excluídos.

$\mathrm{Na}$ Medline foram encontrados 55 artigos dos quais quarenta e seis foram excluídos, isto porque sete não abordavam a temática não farmacológica, doze não possuíam acesso, sendo selecionados para leitura na íntegra nove artigos, destes apenas um foi para a composição da tabela, e oito foram excluídos por não abordar o cenário requerido pela pergunta norteadora, totalizado cinquenta e quatro artigos excluídos. A Scielo teve um total de 10 artigos localizados, destes cinco foram excluídos, pois um não abordava a temática não farmacológica e quatro possuíam inconformidades. Dos cinco selecionados para a leitura na íntegra, três foram selecionados para compor o quadro sendo dois excluídos por não abordarem o deste trabalho, totalizando sete artigos excluídos.

Os 11 artigos analisados a seguir, tratam acerca dos tratamentos não farmacológicos para HAS na Atenção Primária como tema central ou estão envolvidos no contexto da hipertensão em amplo espectro de tratamentos, trazendo a temática como tópico de relevância, por isso foram selecionados para integrar a Tabela 2: 
Tabela 2: Distribuição das produções científicas segundo as seguintes variáveis: o ano de publicação, título, autoria, objetivo e conclusão $(n=11)$.

\begin{tabular}{|c|c|c|c|c|}
\hline ANO & TÍTULO & AUTORIA & OBJETIVO & CONCLUSÃO \\
\hline 2015 & $\begin{array}{l}\text { Análise de três } \\
\text { estratégias de } \\
\text { educação em saúde } \\
\text { para portadores de } \\
\text { hipertensão arterial }\end{array}$ & $\begin{array}{c}\text { Machado, et } \\
\text { al. }\end{array}$ & $\begin{array}{l}\text { Comparar o efeito de três estratégias de } \\
\text { educação em saúde e nutrição sobre a } \\
\text { adesão ao tratamento não } \\
\text { farmacológico da hipertensão arterial } \\
\text { sistêmica (HAS), pelos parâmetros } \\
\text { antropométricos, bioquímicos, clínicos } \\
\text { e dietéticos. }\end{array}$ & $\begin{array}{l}\text { Os Grupo } 1 \text { e } 2 \text { tiveram melhores resultados } \\
\text { sobre a adesão ao tratamento não } \\
\text { farmacológico da HAS. É preciso salientar que } \\
\text { na prática dos serviços de saúde há o desafio } \\
\text { de se promover uma educação em saúde capaz } \\
\text { de intervir sobre a problemática da adesão ao } \\
\text { tratamento. }\end{array}$ \\
\hline
\end{tabular}

Fatores

determinantes da

adesão de

hipertensos a

conduta dietoterápica
O estudo objetivou avaliar os

Massing \& Portella fatores determinantes da adesão de hipertensos a uma conduta dietoterápica.
O estudo permitiu observar o conhecimento dos pacientes quanto ao tratamento não medicamentoso e notou a importância de novas estratégias de controle da hipertensão.
Impacto da estratégia de grupo educativo no melhoramento de 2016 parâmetros clínicos e glicêmicos de diabéticos e hipertensos
Favaro, et al.

Avaliar o impacto da estratégia de grupo educativo no melhoramento de parâmetros clínicos e glicêmicos de diabéticos e hipertensos.
A importância dos resultados sugere que a estratégia de utilizar grupos educativos têm efeito favorável para o controle de diabetes mellitus e da hipertensão

\section{Eficácia da} intervenção multidisciplinar no controle da pressão arterial na atenção primária à saúde: um ensaio clínico randomizado

Kuhmmer, et al.

O objetivo deste estudo é comparar a eficácia de um programa multidisciplinar baseado no atendimento em grupo e individual versus atendimento apenas em grupo, para promover o controle da pressão arterial em pacientes hipertensos na atenção primária à saúde
O estudo demonstra eficácia semelhante de uma intervenção em grupo em comparação a um programa de educação personalizada em pacientes com hipertensão para atingir o controle da PA. Esses achados indicam que a intervenção pode ser para todos os hipertensos atendidos na atenção primária à saúde

\begin{tabular}{|c|c|c|c|c|}
\hline 2017 & $\begin{array}{l}\text { Aconselhamento } \\
\text { sobre modos } \\
\text { saudáveis de vida na } \\
\text { Atenção Primária à } \\
\text { Saúde }\end{array}$ & Toledo, et al. & $\begin{array}{l}\text { Verificar a realização de } \\
\text { aconselhamento sobre modos } \\
\text { saudáveis de vida por profissionais } \\
\text { de saúde da Atenção Primária, } \\
\text { segundo a percepçâo dos usuários, } \\
\text { e fatores associados a esta prática; }\end{array}$ & $\begin{array}{c}\text { O aconselhamento sobre modos saudáveis de } \\
\text { vida precisa ser revisto de forma a promover a } \\
\text { equidade e a integralidade do cuidado em } \\
\text { saúde, tendo em vista o papel e o potencial da } \\
\text { Atenção Primária }\end{array}$ \\
\hline 2017 & $\begin{array}{c}\text { Adesão ao } \\
\text { tratamento da } \\
\text { hipertensão arterial } \\
\text { entre usuários da } \\
\text { estratégia saúde da } \\
\text { família em um } \\
\text { município do Piauí }\end{array}$ & Rocha, et al. & $\begin{array}{c}\text { Este estudo investigou a adesão ao } \\
\text { tratamento da hipertensão arterial } \\
\text { entre usuários da Estratégia Saúde } \\
\text { da Família no município de } \\
\text { Floriano }\end{array}$ & $\begin{array}{c}\text { Esta investigação constitui um diagnóstico } \\
\text { relevante para a compreensão das dificuldades } \\
\text { para adesão ao tratamento e para o adequado } \\
\text { seguimento de terapêutica, contribuindo para } \\
\text { integralidade e resolutividade da atenção à } \\
\text { saúde }\end{array}$ \\
\hline 2017 & $\begin{array}{l}\text { Diretrizes em } \\
\text { hipertensão arterial } \\
\text { para cuidados } \\
\text { primários nos países } \\
\text { de língua portuguesa }\end{array}$ & Oliveira, et al. & $\begin{array}{c}\text { Estabelecer orientações para a } \\
\text { sociedade médica em relação a } \\
\text { Hipertensão Arterial nos Países de } \\
\text { Língua Portuguesa }\end{array}$ & $\begin{array}{l}\text { Torna-se necessário garantir a implementação } \\
\text { das diretrizes para o tratamento da HAS } \\
\text { através de um processo continuado, } \\
\text { envolvendo fundamentalmente ações de } \\
\text { educação, de mudança do estilo de vida e } \\
\text { garantia de acesso aos medicamentos }\end{array}$ \\
\hline
\end{tabular}




\begin{tabular}{|c|c|c|c|c|}
\hline 2018 & $\begin{array}{l}\text { Intervenções não } \\
\text { farmacológicas } \\
\text { associadas à } \\
\text { prevalência e } \\
\text { incidência da } \\
\text { hipertensão arterial: } \\
\text { uma revisão } \\
\text { integrativa }\end{array}$ & Freitas, et al. & $\begin{array}{l}\text { Descrever a associação entre as } \\
\text { medidas não farmacológicas, na } \\
\text { Atenção Primária e Secundária, e a } \\
\text { prevalência e incidência da HAS }\end{array}$ & $\begin{array}{l}\text { O incentivo à adoção de hábitos de vida } \\
\text { saudáveis, em abordagem e acompanhamento } \\
\text { multidisciplinar, componentes indissociáveis } \\
\text { na efetivação do exercício da saúde de } \\
\text { qualidade, demonstrou contribuição na } \\
\text { prevenção da HAS e no tratamento não- } \\
\text { farmacológico do indivíduo hipertenso, no } \\
\text { âmbito da Atenção Primária e Secundária }\end{array}$ \\
\hline 2020 & $\begin{array}{l}\text { Ações específicas } \\
\text { para o controle da } \\
\text { pressão de pulso em } \\
\text { hipertensos e } \\
\quad \text { diabéticos }\end{array}$ & Pelazza, et al. & $\begin{array}{c}\text { Avaliar os parâmetros } \\
\text { hemodinâmicos em homens e } \\
\text { mulheres e elaborar propostas de } \\
\text { ações farmacológicas e não } \\
\text { farmacológicas para pacientes com } \\
\text { pressão de pulso (PP) acima da } \\
\text { média }\end{array}$ & $\begin{array}{c}\text { Evidenciou-se a prevalência do sexo feminino } \\
\text { e o aumento progressivo da PP, o que } \\
\text { representa elevado risco cardiovascular (RCV) } \\
\text { nesta população. Assi, destaca-se a } \\
\text { necessidade de maiores intervenções com } \\
\text { direcionamento específico a este grupo }\end{array}$ \\
\hline 2020 & $\begin{array}{l}\text { Não Adesão ao } \\
\text { Tratamento não } \\
\text { Medicamentoso da } \\
\text { Hipertensão Arterial } \\
\text { Sistêmica }\end{array}$ & Macete \& Borges & $\begin{array}{l}\text { Verificar como os fatores estão } \\
\text { associados à não adesão ao } \\
\text { tratamento não medicamentoso da } \\
\text { Hipertensão Arterial Sistêmica }\end{array}$ & $\begin{array}{l}\text { O indivíduo deve ser protagonista em todo } \\
\text { processo para a tomada de decisões. Acredita- } \\
\text { se num processo de educação em saúde, capaz } \\
\text { de conduzir a pessoa hipertensa à adesão às } \\
\text { condutas de controle da HAS através de } \\
\text { estratégias com foco nos fatores modificáveis }\end{array}$ \\
\hline 2020 & $\begin{array}{l}\text { Eficácia de um } \\
\text { programa abrangente } \\
\text { de controle da } \\
\text { pressão arterial na } \\
\text { atenção primária à } \\
\text { saúde no Peru }\end{array}$ & Kim, et al. & $\begin{array}{l}\text { Avaliar a eficácia de um programa } \\
\text { abrangente de controle da pressão } \\
\text { arterial (PA) na melhoria de } \\
\text { indicadores do estilo de vida, dos } \\
\text { níveis de PA e dos resultados de } \\
\text { exame laboratoriais entre pessoas } \\
\text { que vivem em áreas urbanas de } \\
\text { baixa renda no Peru }\end{array}$ & $\begin{array}{l}\text { Este programa abrangente de controle de PA } \\
\text { foi eficaz na melhoria de indicadores do estilo } \\
\text { de vida, dos níveis de PA e dos resultados } \\
\text { laboratoriais entre pessoas que vivem em uma } \\
\text { área urbana de baixa renda no Peru. O } \\
\text { aumento das oportunidades para mediação da } \\
\text { PA, a gestão sistemática de pacientes } \\
\text { hipertensos e programas comunitários de } \\
\text { prevenção e educação são fundamentais para } \\
\text { detectar, prevenir e controlar a hipertensão }\end{array}$ \\
\hline
\end{tabular}

Fonte: Autores.

O diagnóstico de HAS traz muitas implicações para a vida do paciente, ainda que, em um primeiro momento o mesmo não internalize as repercussões que isto terá em sua vida. Ao longo do processo de adoecimento, quando não controlada, as complicações acabam por fazer evidente a relevância que tais implicações possuem. As LOA por exemplo, configuram-se como uma dessas complicações. Uma forma que está se mostrando eficiente para prevenir e tratar que intercorrências decorrentes da HAS, sobretudo em pessoas que possuem comorbidades, são as mudanças nos hábitos de vida. As quais minimizam os fatores de risco modificáveis e de acordo com Oliveira et al. (2017) as referidas ações são mais promissoras no ambiente da Atenção Básica. Estas representam, na maioria das vezes, restrições de prazeres da vida e alterações bruscas na rotina, portanto não se tornam medidas fáceis de serem aceitas e implantadas no cotidiano dos pacientes.

Os hábitos de vida incluem um extenso rol de práticas que podem ser modificadas afim de trazer melhorias para a saúde do indivíduo. Dentre estas, pode-se elencar: atividade física, tabagismo, etilismo, relações interpessoais, inteligência emocional, medidas antropométricas e nutricionais. Neste último há uma infinidade de modificações que podem ser feitas, como redução de sódio, lipídios, alimentos não protetores, bebidas gaseificadas, açúcares, entre outros. O sentimento que envolve ser portador de uma patologia crônica tem uma representatividade muito inquietante para o paciente, dessa forma os fatores culturais acabam influenciando em como o indivíduo vai enfrentar a transição, ou não, no modo de agir compatível com o seu atual contexto de saúde. As mudanças de hábitos são estratégias de educação no âmbito da saúde que corroboram com o desenvolvimento de uma sensibilização necessária para se obter uma maior estimativa e qualidade de vida, isto porque estudos 
já confirmaram que problemas como obesidade, sedentarismo e estresse aumentam o surgimento de HAS e todas as consequências que esta acarreta (Massing \& Portella, 2015; Machado et al., 2015; Pelazza et al., 2020).

O sedentarismo é apontado como a causa número um do aumento da incidência de várias doenças, incluindo a HA. De fato, todos os artigos são unanimes quanto aos benefícios que a atividade física agrega a saúde, para Freitas et al. (2018) entre as práticas não farmacológicas esta é uma das que possui o maior impacto na redução da PA e na melhoria da qualidade de vida, conseguindo influenciar de forma benéfica em todos os sistemas corpóreos. Isto fica evidente quando se avalia modificações nas medidas antropométricas, como a circunferência abdominal e Índice de Massa Corporal (IMC), que são importantes preditivos de risco cardiovascular, os quais podem ser melhorados por meio dos exercícios, assim estes se configuram como um importante aliado do controle dos níveis pressóricos. A atividade regular para esses pacientes é definida como aquela realizada em pelo menos cinco dias na semana, com duração mínima de trinta minutos e intensidade moderada (Massing \& Portella, 2015; Rocha et al., 2017).

A dietoterapia tem destaque no âmbito do tratamento não farmacológico, uma vez que, os alimentos assumem ação positiva ou negativa no organismo, a depender da sua constituição nutricional e da quantidade ingerida. Massing e Portella (2015) afirmam que as mudanças nutricionais podem ser uma causa de não persistência no tratamento, isto porque alguns alimentos com carga nutricional saudável, tais como fibras, hortaliças, frutas, verduras, carboidratos complexos, proteínas, baixo teor de gordura, leites e derivados desnatados, podem não ser a princípio palatável pra quem não possui o hábito de consumi-los. As mudanças dietéticas também visam adequação do peso e devem ser feitas por meios de planos alimentares aplicados de acordo com as particularidades de cada paciente, as quais associadas as atividades físicas, constituem pilares para uma vida mais saudável.

Dentro das mudanças nutricionais, a redução do uso de sódio, comumente consumido por meio do sal de cozinha, tem um papel de destaque. A VII Diretriz Brasileira de Hipertensão e a Organização Mundial de Saúde, determinam que o uso do sal é benéfico para o organismo, no entanto no Brasil o uso corresponde ao dobro do que é sugerido - no máximo $2 \mathrm{~g}$ de sódio por pessoa diariamente. Os estudos indicam que a redução no consumo deste componente tem implicação direta nos valores pressóricos, obtendo resultados rápidos e significativos. Vale ressaltar que esta é uma medida bem consolidada no conhecimento popular (Massing \& Portella, 2015). De acordo com Kim et al. (2020) demonstrou em seu estudo que pequenas mudanças como introdução de frutas e vegetais, bem como a redução do sódio conseguiram ter repercussões positivas na redução da PA sistólica e diastólica, esses hábitos associados as demais mudanças do estilo de vida conseguem refletir em benefícios que extrapolam o sistema cardíaco e circulatório.

A Diretriz Canadense (2020), trouxe a partir da versão do ano de 2016, algumas mudanças, e entre elas está o aumento do uso de potássio na dieta para indivíduos que não possuam risco significativo de hipercalemia. No Brasil alguns estudos já apontavam o uso do potássio, do cálcio e do magnésio em quantidades adequadas na alimentação como compostos químicos no auxílio do tratamento e prevenção da HAS. Destaca-se que todos esses componentes podem ser obtidos por meio de uma alimentação balanceada. Esta estratégia de tratamento através da educação e nutrição refletem em melhores resultados nos parâmetros antropométricos, bioquímicos e dietéticos. No entanto o acompanhamento e continuidade por meio do apoio profissional, são necessários para que as mudanças sejam incorporadas ao estilo de vida a médio e longo prazo (Massing \& Portella, 2015; Machado et al., 2015).

O exercício físico e a dietoterapia contribuem em conjunto para um dos principais objetivos do tratamento não farmacológico: a perda do excesso de peso. Esta mudança tem um efeito positivo na regulação da pressão arterial e no metabolismo do indivíduo. No estudo epidemiológico transversal de Massing e Portella (2015) envolvendo 12.324 adultos, evidenciou-se que o número de hipertensos descompensados, foi menor entre aqueles que tiveram orientação adequada para manter ou atingir seu peso ideal, comparado aos que apenas realizaram o eletrocardiograma. Os artigos determinam que a 
perda de dez quilos da massa corpórea pode reduzir a pressão arterial de 5 a $20 \mathrm{mmHg}$, tornando-se uma intervenção não farmacológica indispensável, bem como indissociável à prática de atividade física e dieta (Machado et al., 2015).

O uso de droga lícitas compõem costumes que devem ser reavaliados. No que diz respeito ao tabagismo, é preconizado a suspensão do hábito, afim de evitar os riscos para HAS, os quais aumentam na proporção da quantidade de cigarros utilizados. Ressalta-se que as mulheres fumantes apresentam maior risco de complicações cardiovasculares. A relação do álcool com a elevação da PA é clara, o que desencadeia maiores comorbidades, deste modo existem indicações especificas de ingestão diária e/ou semanal, diferenciadas para homens e mulheres. Estas indicações possuem variações na forma de apresentação, dependendo da literatura as referências estão em parâmetros de gramas, doses ou drinks. Na Diretriz Brasileira, utilizada como parâmetro para todos os trabalhos aqui analisados, a recomendação é de 1 dose para mulheres e 2 doses para homens ( 1 dose corresponde aproximadamente a $14 \mathrm{~g}$ de álcool). Na diretriz canadense a recomendação é de uma quantidade inferior a 14 drinques para homens e a 9 para mulheres. Estudos relatam ainda que o fato de ingerir álcool está relacionado com maiores chances de não adesão ao tratamento da HAS, o que chama atenção para o consumo dessas drogas lícitas (Massing \& Portella, 2015; Malachias, 2016; Hypertension Highlights, 2020; Rocha, et al., 2017).

O tratamento não farmacológico pode ser implantado a princípio em todos os estágios da HAS, mas se mostram mais eficaz em pacientes pré-hipertensos ou com hipertensão classe I e com risco cardiovascular moderado ou baixo, muitas vezes, é o único tratamento realizado nesses casos (Oliveira, et al., 2017). O Canadá caracteriza-se como uma referência mundial por ter os melhores dados epidemiológicos referentes a HAS, sua diretriz apresenta algumas modificações quando comparada com a brasileira, que podem ter sido determinantes no alcance de um quantitativo modelo para os demais países. Nesta perspectiva, algumas das mudanças observadas na sua Guideline diz respeito ao modo como ocorre a aferição da PA, que se dá por meio automatizado, com o paciente sozinho e fazendo médias dos resultados obtidos. Ademais disponibiliza um acompanhamento continuado. No que diz respeito as orientações não medicamentosas, compartilha quase que integralmente das orientações disponibilizadas na Diretriz Brasileira. (Hypertension Highlights, 2020; Malachias et al., 2017)

O tratamento continuado toma relevância quando se entende que a terapia não medicamentosa é mais difícil por requerer mudanças no dia-a-dia, deste modo, o apoio dos profissionais da saúde se faz determinante para o sucesso do tratamento, com avaliações periódicas e auxilio a nova realidade. Outrossim, é sempre importante ressaltar onde estão as maiores dificuldades para o sucesso deste método: baixa escolaridade, poder aquisitivo reduzido, não aceitação das mudanças de hábitos de vida e o fato de ser uma doença assintomática. Todo esse panorama torna-se obstáculo para adesão, uma vez que dificulta o acesso as formas alternativas de tratamento, resultando em exacerbação dos fatores que geram complicações. (Favaro et al., 2016; Macete \& Borges, 2020).

O tratamento não medicamentoso sem dúvida tem capacidade de influenciar beneficamente no processo de estabilização dos níveis pressóricos, entretanto um entrave para que ele se torne efetivo é a adesão dos pacientes, visto que o papel da aceitação deste é primordial. Kuhmmer et al. (2016), traz a perspectiva de que a benesse de um programa multidisciplinar é decisiva para o sucesso na adesão as mudanças nos diversos âmbitos dos hábitos de vida, e até mesmo no tratamento medicamentoso, quando há a necessidade deste ser implantado. Ademais, as orientações podem ser repassadas em grupo com a mesma eficácia de um atendimento personalizado, uma vez que a continuidade do acompanhamento é que faz o sucesso na adesão.

Entre as objeções colocadas pelos pacientes está a dificuldade nas mudanças alimentares, criação de novos hábitos menos prazerosos, demora no agendamento de consultas, falta de apoio e fatores socioeconômicos. Além disso, o paciente parece se isentar do seu papel no controle da PA, e o fato de não seguir as orientações que lhe são passadas reflete diretamente nos desdobramentos desse processo de adoecimento, apesar de que muitas das medidas necessárias não são desconhecidas 
pelos pacientes. O fato de ser uma patologia com manifestações silenciosas diminui o impacto da necessidade de mudanças até que surjam sintomas alarmantes. (Massing \& Portella, 2015).

O papel do profissional da saúde neste tratamento, não é apenas ensinar os métodos não farmacológicos de controle da PA, até porque, como já mencionado, muitos pacientes já conhecem grande parte deles, mas sim orientar sobre os desdobramentos da necessidade de ter que se iniciar um tratamento medicamentoso com seus respectivos efeitos colaterais, rigorosidade na ingestão das capsulas e associação de medicamentos. Aquele também se torna primordial ao incitar as mudanças de hábitos com uma postura ativa do indivíduo no controle da sua doença por meio de intervenções periódicas e continuas. Para isso, a forma como o profissional repassa as informações é crucial, possuindo empatia, trabalho multidisciplinar, comunicação e, sobretudo, ouvir as dificuldades do paciente, não impondo, mas aconselhando e mostrando as melhores alternativas. Esse conjunto de fatores são indispensáveis para manter a continuidade na adesão ao tratamento. (Massing \& Portella, 2015; Freitas et al., 2018; Favaro et al., 2016; Toledo et al., 2017).

\section{Conclusão}

Conforme demonstrado as mudanças nos hábitos de vida tem efeitos positivos e consideráveis no tratamento não medicamentoso da HAS, e estas podem ser inseridas e estimuladas por meio de um acompanhamento continuado rigoroso. Quando se fala em continuado, não implica dizer que é diário, mas um acompanhamento que seja mantido na estratégia de cuidado desses pacientes, onde exista sempre a preocupação em determinados períodos de procurar este grupo para avaliar como está a implantação e adaptação de novos costumes.

A Atenção Primária tem as ferramentas necessárias para que esse acompanhamento possa ocorrer, as visitas domiciliares mensais, rodas de conversas nas Unidades Básicas de Saúde e os questionamentos nas consultas de rotina, podem ser usados como instrumento de sensibilização dos meios práticos para o controle dos níveis pressóricos de acordo com a realidade de cada paciente, o que facilitaria a implementação dessa forma de tratamento com o melhor custo benefício para o paciente e o sistema de saúde.

Trabalhos que abordem esse tema se tornam relevante pelo caráter preventivo que podem possuir, reduzindo custos e disponibilizando oferta e demanda para outras necessidades que não possuem a possibilidade de educação em saúde. Assim, trabalhos futuros podem contabilizar o impacto que as complicações geram no sistema de saúde e o quanto poderia ser destinado pra melhorias do sistema de saúde, caso as medidas de hábitos de vida fossem assimiladas na rotina diária.

\section{Agradecimentos}

Agradecemos primeiramente a Deus por ter permitido em um ano de tantos desafios vencermos mais um, e publicar este artigo, o que nos trouxe imensa alegria e gratidão. Agradecemos a Ele também por ter nos reunido, e assim podermos desenvolver este trabalho que nos dá imensa satisfação. Somos muito gratos aos nossos familiares, pelo apoio e compreensão dos dias ausentes na jornada do curso e da vida científica. E por fim, mas não menos importante, somos imensuravelmente gratos as nossas orientadoras que nos fornecem tanto apoio acadêmico, repassando suas experiências e conhecimentos, sendo solícitas em dias e horários extras, nos incentivando a sempre ir mais longe e nunca cessar a busca pelo conhecimento. Nosso muito obrigado a todos!

\section{Referências}

Brasil. (2013). Departamento de Informática do SUS. Hiperdia - Sistema de Cadastramento e Acompanhamento de Hipertensos e Diabéticos. Ministério da Saúde. http://tabnet.datasus.gov.br/cgi/deftohtm.exe?hiperdia/cnv/hdMA.def 
Canadá (2020). Hypertension Highlights (Guideline). Hypertension Canada. https://hypertension.ca/wp-content/uploads/2018/07/Hypertension-GuidelinesEnglish-2018-Web.pdf

Consolim-Colombo, F. M., \& Saraiva, J. F. K., \& Izar, M. C. O. (2019). Tratado de Cardiologia: SOCESP. editora Manole.

Dantas, R. C. O., \& Roncalli, A. G. (2019). Protocolo para indivíduos hipertensos assistidos na Atenção Básica em Saúde. Ciência \& Saúde Coletiva, 24(1), 295-306.

Favaro, D. T. L. et al. (2016). Impact of educational group strategy to improve clinical and glycemic parameters in individuals with diabetes and hypertension. Investigación y Educación en Enfermería, 34(2), 314-321.

Fiório, C. E. et al. (2020). Prevalência de hipertensão arterial em adultos no município de São Paulo e fatores associados. Revista Brasileira de Epidemiologia, 23.

Freitas, M. M. A. et al. (2018). Intervenções não-farmacológicas associadas à prevalência e incidência da hipertensão arterial: uma revisão integrativa. Fisioterapia Brasil, 19 (2), 265-271.

Gewehr, D. M. et al. (2018). Adesão ao tratamento farmacológico da hipertensão arterial na Atenção Primária à Saúde. Saúde em Debate, 42(116), 179-190.

Jardim, P. C. B. V. (2018). A SBC e a Hipertensão Arterial: É Hora de Ação. Arquivos Brasileiros de Cardiologia, 111(3), 343-344.

Kim, J. K. et al. (2020). Effectiveness of a comprehensive blood pressure control program in primary health care in Peru. Revista Panam Salud Publica, 44(18), 1-8.

Kuhmmer, R. et al. (2016). Effectiveness of multidisciplinary intervention on blood pressure control in primary health care: a randomized clinical trial. $B M C$ health services research, 16 (1), 1-13.

Laboissière, P. (2019). Hipertensão afeta um em cada quatro brasileiros adultos. Agência Brasil. https://agenciabrasil.ebc.com.br/saude/noticia/201904/hipertensao-afeta-um-em-cada-quatro-brasileiros-adultos.

Macete, K. G. \& Borges, G. F. (2020). Não Adesão ao Tratamento não Medicamentoso da Hipertensão Arterial Sistêmica. Saúde em Foco, 7(1), 128-154.

Machado, J. C. et al. (2016). Análise de três estratégias de educação em saúde para portadores de hipertensão arterial. Ciência \& Saúde Coletiva, 21(2), 611620.ver o ano.

Malachias, M. V. B. et al. (2016). $7^{\text {a }}$ Diretriz Brasileira de Hipertensão Arterial. Arquivos Brasileiros de Cardiologia, 107(3), 1-103.

Malachias, M. V. B. et al. (2017). $7^{\text {a }}$ Diretriz Brasileira de Hipertensão Arterial. Revista Brasileira de Hipertensão, $24(1), 1-91$.

Malta, D. C. et al. (2018). Prevalência da hipertensão arterial segundo diferentes critérios diagnósticos, Pesquisa Nacional de Saúde. Revista Brasileira de Epidemiologia, 28 (1).

Massing, L. T. \& Portella, M. R. (2015). Fatores determinantes da adesão de hipertensos a conduta dietoterápica. Revista de Atenção à Saúde, $13(43), 37-45$.

Oliveira, B. L. C. A. et al. (2020). A influência da Estratégia Saúde da Família no uso de serviços de saúde por adultos hipertensos no Brasil. Revista Brasileira de Epidemiologia, 23.

Oliveira, G. M. M. et al. (2017). 2017: Diretrizes em Hipertensão Arterial para Cuidados Primários nos Países de Língua Portuguesa. Arquivos Brasileiros de Cardiologia, 109(5), 389-396.

Organização Pan-Americana da Saúde (2020). Doenças cardiovasculares. https://www.paho.org/bra/index.php?option=com_content\&view=article\&id=52 53:doencas-cardiovasculares\&Itemid=1096.

Pelazza, B. B. et al. (2020). Ações específicas para o controle da pressão de pulso em hipertensos e diabéticos. Revista Nursing, $23(261)$, 3594-3599.

Rocha, M. L., \& Borges, J. W., \& Martins, M. F. S. (2017). Adesão ao tratamento da hipertensão arterial entre usuários da estratégia saúde da família em um município do Piauí. Revista de APS, 20(1), 6-20.

Santiago, E. R. C. et al. (2019). Prevalência e Fatores Associados à Hipertensão Arterial Sistêmica em Adultos do Sertão de Pernambuco, Brasil. Arquivos Brasileiros de Cardiologia, 113(4), 687-695.

Scholze, A. S. et al. (2019). Hipertensão Arterial Sistêmica: a Perspectiva dos Docentes no Ensino Médico. Revista Brasileira de Educação Médica, 43(4), 8291.

Toledo, M. T. T et al. (2017). Aconselhamento sobre modos saudáveis de vida na Atenção Primária à Saúde. O Mundo da Saúde, 41 (1), 87-97. 